\title{
Conformational Rigidity within Plasticity Promotes Differential Target Recognition of Nerve Growth Factor
}

\author{
Francesca Paoletti ${ }^{1,2}$, Cesira de Chiara ${ }^{3}$, Geoff Kelly ${ }^{4}$, Sonia Covaceuszach ${ }^{5}$, \\ Francesca Malerba ${ }^{1,2}$, Robert Yan ${ }^{6}$, Doriano Lamba ${ }^{5}$, Antonino Cattaneo ${ }^{1,2 *}$ and \\ Annalisa Pastore ${ }^{6,7 *}$
}

${ }^{1}$ Neurotrophic Factors and Neurodegenerative Diseases Unit, European Brain Research, Rita Levi-Montalcini Foundation, Rome, Italy, ${ }^{2}$ Scuola Normale Superiore, Pisa, Italy, ${ }^{3}$ The Francis Crick Institute, London, UK, ${ }^{4}$ Medical Research Council (MRC) Biomedical NMR Centre, The Francis Crick Institute, London, UK, ${ }^{5}$ Istituto di Cristallografia, Consiglio Nazionale delle Ricerche (CNR), Sede Secondaria di Basovizza, Trieste, Italy, ${ }^{6}$ Maurice Wohl Institute, Department of Basic and Clinical Neuroscience, King's College London, London, UK, ${ }^{7}$ Molecular Medicine Department, University of Pavia, Pavia, Italy

Nerve Growth Factor (NGF), the prototype of the neurotrophin family, is essential for maintenance and growth of different neuronal populations. The X-ray crystal structure of NGF has been known since the early ' 90 s and shows a $\beta$-sandwich fold with extensive loops that are involved in the interaction with its binding partners. Understanding the dynamical properties of these loops is thus important for molecular recognition. We present here a combined solution NMR/molecular dynamics study which addresses the question of whether and how much the long loops of NGF are flexible and describes the N-terminal intrinsic conformational tendency of the unbound NGF molecule. NMR titration experiments allowed identification of a previously undetected epitope of the anti-NGF antagonist antibody $\alpha \mathrm{D} 11$ which will be of crucial importance for future drug lead discovery. The present study thus recapitulates all the available structural information and unveils the conformational versatility of the relatively rigid NGF loops upon functional ligand binding.

Keywords: antibody recognition, NGF, neurodegeneration, neurotrophins, NMR, structure

\section{INTRODUCTION}

Nerve Growth Factor (NGF), the prototype of the neurotrophin family, is essential for maintenance and growth of different neuronal populations in the nervous systems (Levi-Montalcini, 1987). Alterations in its homeostatic regulation are involved in severe pathologies (Tiveron et al., 2013; Mysona et al., 2015). NGF is translated as a precursor, proNGF, with distinct biological functions (Hempstead, 2014). An early event in NGF signal transduction is the interaction with p $75^{\mathrm{NTR}}$ and/or TrkA receptors (Lewin and Carter, 2014). Because of its role in the pathway of pain (Capsoni, 2014; Kelleher et al., 2017), there is a substantial interest in developing NGF mimetics endowed with antagonistic properties. However, this strongly relies on a detailed knowledge of its structure and of the interactions with its receptors.

The X-ray crystal structures of NGF have been known since the early 90s (McDonald et al., 1991; Holland et al., 1994). NGF is an obligate non-covalent head-to-head homodimer with three loops (I, II, and V) at one end of the molecule, whereas the opposite end contains a loop structure (III) and 
the conserved cystine-knot arrangement of the three intrachain disulphide bonds. A distinctive feature of NGF are its long unstructured loops (I, II, III, and V), important for its interactions with the binding partners.

Also available are the structures of NGF bound to its receptors (Wiesmann et al., 1999; He and Garcia, 2004; Wehrman et al., 2007; Feng et al., 2010), neutralizing antibodies (Covaceuszach et al., 2008; La Porte et al., 2014), and small ligands, such as lysophospholipids (Tong et al., 2012; Sun and Jiang, 2015) and DNA-aptamers (Jarvis et al., 2015). These studies have led to an emerging picture of the binding determinants on NGF to its key receptors.

Despite the wealth of structural information obtained from the extensive crystallographic investigations, some significant questions have remained elusive: (i) is the NGF N-terminus unstructured in the absence of ligands or does it already have some intrinsic conformational tendency? (ii) how flexible/rigid are the loops and how their dynamics may reflect on the overall structural plasticity of mature NGF? (iii) how much do the loops contribute to antibody recognition?

Structural information is thus extremely important, in view of structure-based design of effective non-peptidic antagonists of NGF activity on TrkA receptor. Accumulating evidence shows that the NGF N-terminus plays a role in a number of processes. An N-terminal truncated form of NGF has a significant drop in affinity for TrkA and in the ability of eliciting TrkA phosphorylation (Kahle et al., 1992; Hughes et al., 2001) which could not be ascribed to differences in folding or stability (Woo et al., 1995). In one of the two crystal structures of NGF purified from mouse salivary glands (mNGF), the $\mathrm{N}$-terminus is absent (Holland et al., 1994) whereas in the other neither $\mathrm{N}$ - nor C- termini were defined (McDonald et al., 1991). The N-terminus in recombinant human NGF (hNGF) or mouse proNGF in complex with p75 ${ }^{\mathrm{NTR}}$ are not defined (He and Garcia, 2004; Feng et al., 2010). Only in the hNGF/TrkA complex (Wiesmann et al., 1999; Wehrman et al., 2007) and in one of the two protomers of a complex with a DNA aptamer (Jarvis et al., 2015), is the $\mathrm{N}$-terminus defined and folded in an $\alpha$-helix. The generally accepted view is thus that the $\mathrm{N}$-terminus is disordered in the NGF unbound state (Settanni et al., 2003; Berrera et al., 2006). Similar conclusions have been recently reached as result of CD and NMR solution studies and molecular dynamics simulations on two linear hNGF N-terminus peptides (Stanzione et al., 2010; Travaglia et al., 2015).

It is difficult to infer from the X-ray data whether the variations reflect authentic flexibility or rather a structural plasticity. The two terms are similar but not equivalent: In the context of a protein, we would consider plasticity the capacity of a region to change the conformation permanently under, for instance, an interaction; flexibility is the property of a region (e.g., a loop) not to have a fixed conformation. In other words, one is a static concept, the second dynamic. The difference is particularly important for the design of antibodies directed against specific regions of NGF. Among the existing anti-NGF antibodies, the well-characterized therapeutic MAb $\alpha \mathrm{D} 11$ (Cattaneo et al., 1988; Covaceuszach et al., 2012) is particularly interesting as a structural probe, because it recognizes preferentially the mature form of NGF vs. proNGF (Paoletti et al., 2009). This property has been used to study the mechanistic consequences of inducing experimentally an imbalance in NGF/proNGF (Capsoni and Cattaneo, 2006; Capsoni et al., 2011). The MAb $\alpha$ D11 epitope has been characterized by ELISA, and the structure of the complex of rat $\alpha \mathrm{D} 11 \mathrm{FAb}$ and hNGF was obtained by in silico computational docking and validated by SAXS experiments (Covaceuszach et al., 2008).

We present here a study in solution which addresses the open questions. Using a combination of nuclear magnetic resonance (NMR) and molecular dynamics (MD), we describe the N-terminal intrinsic conformational preferences of unbound NGF in solution. We also show that in the absence of partners the NGF N-terminus has a strong tendency to fold into a helix, challenging the current view that this region is unstructured. Our study also sets a definitive word on the structural plasticity of NGF loops II and V and provides a structural explanation for the large differential affinity of the $\alpha \mathrm{D} 11$ anti-NGF therapeutic antibody for NGF vs. proNGF. We demonstrate by solution NMR epitope mapping with the MAb $\alpha \mathrm{D} 11$ the presence of a previously undetected epitope. The present study thus fills a gap in our structural understanding of NGF inter- and intramolecular interactions and provides a strong basis for the design of more selective NGF antagonists.

\section{RESULTS}

\section{Solution NMR Structure of Mouse NGF}

Assigning the NMR spectrum to the specific protons of a protein is the prerequisite to map interactions and any conformational change. At $30^{\circ} \mathrm{C}$, the $2 \mathrm{D}^{1} \mathrm{H}-{ }^{15} \mathrm{~N}$ HSQC of $\mathrm{mNGF}$ is optimal and reveals a wide dispersion of signals characteristic of proteins with a predominantly $\beta$-sheet content which is consistent with the $\mathrm{X}$ ray structure. The indole correlations of the three Trp residues are clearly observable in the distinctive and typically isolated region of the spectrum around $10.5 \mathrm{ppm}\left({ }^{1} \mathrm{H}\right)$ and $135 \mathrm{ppm}$ $\left({ }^{15} \mathrm{~N}\right)$. All Gln and Asn side chains are detected. We achieved virtually full assignment of the spectrum. Conversion of the NOE information into a structural model was not trivial because the potential symmetry (two-fold) of the homodimer makes it hard to distinguish between intra- and inter-molecular NOEs. The problem, which has been debated for years (Saudek et al., 1991; Nilges and O'Donoghue, 1998), was circumvented thanks to the software support of ARIA (Rieping et al., 2007) which allows discrimination of intra- from inter-molecular NOEs and careful iterative analysis of the violations (Table $\mathbf{1}$ ). The process led eventually to a well-converged ensemble (Figure 1A) with a root mean square deviation (r.m.s.d.) of $1.3 \AA$ from the structure with the lowest global energy as calculated on 236 residues (Figure 1B). It closely resembles the available X-ray structures, especially in the Cys-knot, while exhibiting larger variability in the loop regions (Figure 1C). The r.m.s.d. values between the structure with the lowest global energy and the crystal structures of unbound mNGF (PDB ID 1BTG, protomers B,C) or NGF bound to lipids (PDB ID 4EAX or 4XPJ) for the backbone atoms of the core residues $(15-22,51-58,78-89,100-111)$ which exclude the loops are $2.32,2.14$, and $2.12 \AA$ respectively. The 
TABLE 1 | NMR refinement statistics of NGF.

\begin{tabular}{|c|c|}
\hline \multicolumn{2}{|l|}{ COMPLETENESS OF RESONANCE ASSIGNMENTS } \\
\hline Backbone (\%) & 97.3 \\
\hline Side Chains (\%) & 72.9 \\
\hline \multicolumn{2}{|l|}{ NMR DISTANCE AND DIHEDRAL RESTRAINTS } \\
\hline \multicolumn{2}{|l|}{ Distance restraints } \\
\hline Total NOEs (used during calculations - per protomer) & 2327 \\
\hline Unambiguous & 2315 \\
\hline Ambiguous & 12 \\
\hline Merged & 4331 \\
\hline Intra-residue ${ }^{a}$ & 1346 \\
\hline Inter-residue ${ }^{\mathrm{a}}$ & 969 \\
\hline Sequential $(|i-j|=1)$ & 421 \\
\hline Non-sequential $(|i-j|>1)$ & 548 \\
\hline Hydrogen bonds (per protomer) & 46 \\
\hline No. of Noe restraints per residue & 19.9 \\
\hline \multicolumn{2}{|l|}{ Total dihedral angle restraints ${ }^{\mathrm{b}}$ (per protomer) } \\
\hline Phi angles & 55 \\
\hline Psi angles & 56 \\
\hline Inter-protomer restraints (per protomer) & 33 \\
\hline \multicolumn{2}{|l|}{ STRUCTURE STATISTICS } \\
\hline \multicolumn{2}{|l|}{ Violations } \\
\hline Distance constraints (>0.3 $)$ & 5 \\
\hline \multicolumn{2}{|l|}{ Deviations from idealized geometry (over 60 structures) } \\
\hline Bond lengths $(\AA)$ & $0.0035 \pm 0.00009$ \\
\hline Bond Angles $\left({ }^{\circ}\right)$ & $0.47 \pm 0.016$ \\
\hline Improper Angles $\left(^{\circ}\right)$ & $1.46 \pm 0.13$ \\
\hline \multicolumn{2}{|l|}{$\begin{array}{l}\text { Ensemble RMSD (over } 60 \text { structures in the refinement } \\
\text { process) }\end{array}$} \\
\hline \multicolumn{2}{|l|}{ All residues } \\
\hline Backbone $(\mathrm{N}, \mathrm{CA}, \mathrm{C}, \mathrm{O})(\AA)$ & $2.58 \pm 1.18$ \\
\hline Heavy atoms $(\AA)$ & $2.89 \pm 1.11$ \\
\hline \multicolumn{2}{|l|}{ Ordered residues $^{\mathrm{C}}$} \\
\hline Backbone $(\mathrm{N}, \mathrm{CA}, \mathrm{C}, \mathrm{O})(\AA)$ & $1.44 \pm 0.64$ \\
\hline Heavy atoms $(\AA)$ & $1.77 \pm 0.57$ \\
\hline
\end{tabular}

a Statistics among unambiguous restraints.

${ }^{b}$ TALOS-derived dihedral restraints.

${ }^{c}$ Ordered residues (for $A$ and $B$ protomers): 5-6, 14, 17-24, 27-38, 41-43, 47-48, 53-58, 63, 67, 70-71, 75-82, 84-92, 97-112, 114.

structure in solution allows us to address a number of crucial aspects as detailed in the following sections.

\section{The Structure of the NGF N-Terminus}

From our data, we can obtain direct information on the structure of the N-terminus (residues S1-M9) from the NOE patterns. In solution, the $\mathrm{N}$-terminus, whose resonances can be observed distinctly, does not adopt a regular secondary structure. However, a number of medium-range NOEs were observed between the backbone and side-chains of these residues, suggesting the existence of a short nascent helical turn (residues 6-10). The average $\mathrm{O}_{i} \ldots \mathrm{NH}_{\mathrm{i}+3}$ and $\mathrm{O}_{\mathrm{i}} \ldots \mathrm{NH}_{\mathrm{i}+4}$ distances between residues S2-G10 were measured for the 20 lowest global energy models to check the presence of distances compatible with hydrogen bonds. Overall, the V6-G10 segment has a clear propensity to form a short helix, the $\mathrm{O}_{\mathrm{i}} \ldots \mathrm{NH}_{\mathrm{i}+3}$ and $\mathrm{O}_{\mathrm{i}} \ldots \mathrm{NH}_{\mathrm{i}+4}$ distances being within

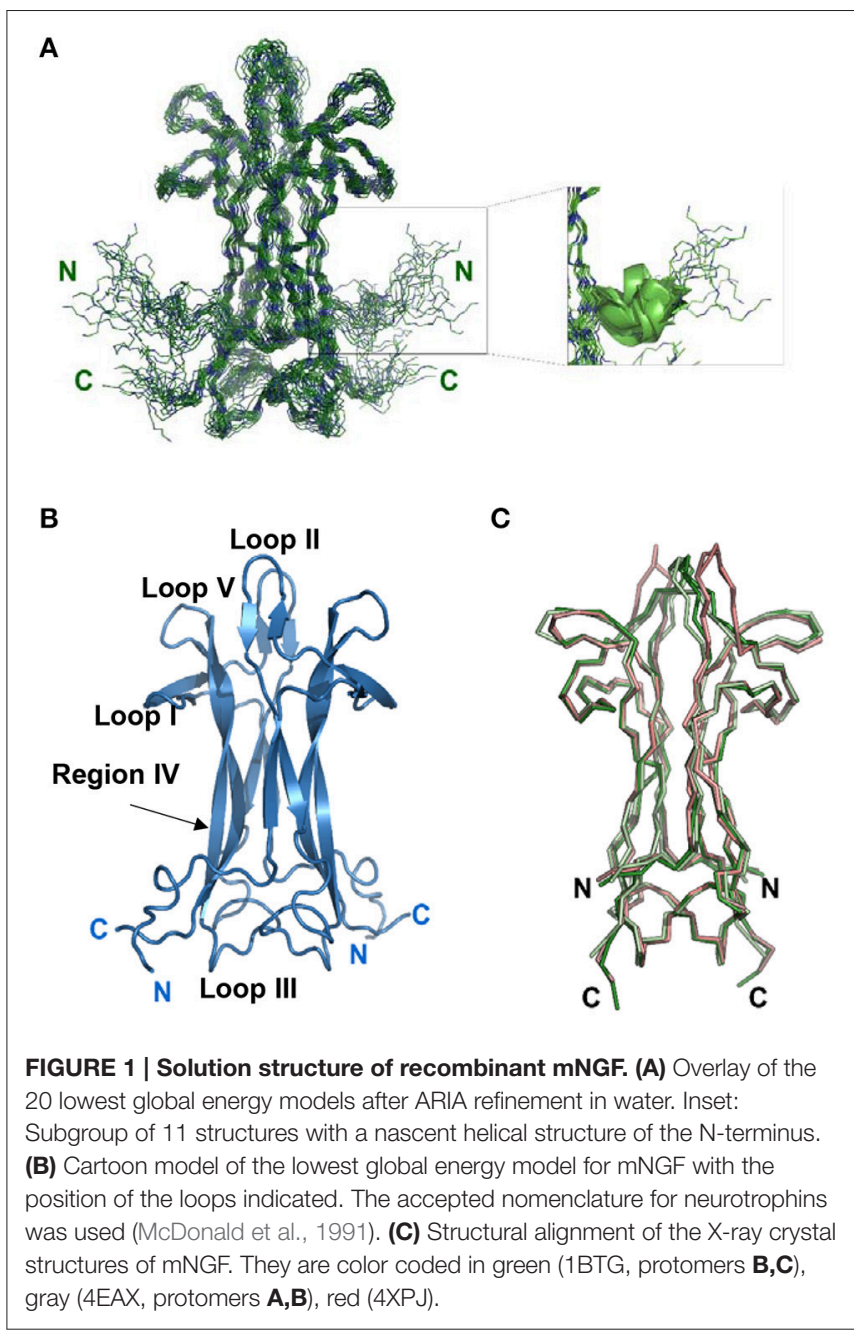

2.6 and $6.1 \AA$ over the whole bundle. In almost $50 \%$ of the 20 lowest global energy models, the V6-G10 segment adopts either a $3_{10}$ helix or a $\alpha$-helix conformation (Figure 1A, inset). These distances are within 2.1 and $6.2 \AA$ in the lower global energy model exhibiting a helical conformation. These results provide a clear answer to whether and how much the N-terminus is folded and clarify its tendency to fold into a helical conformation. The secondary chemical shifts, that is the difference between the observed values and the random coil values for the same amino acid, confirm a helical tendency of this region (data not shown).

\section{The NGF Loops Are in a Slow Conformational Exchange}

While assigning the NGF spectrum which was troublesome due to significant overlap, it became clear that some interesting dynamical processes were going on. At $30^{\circ} \mathrm{C}$ the vast majority of the peaks have homogeneous intensity but a limited set of resonances of weaker intensity are observed (Figure 2A). After virtually full spectral assignment, these weaker resonances were found to be duplicate assignments for a small set of specific resonances which cluster around loops I and II and on the 
stem region (region $\mathrm{IV}$-loop $\mathrm{V}$ ) that points toward loop II (residues 39-47) (Figures 2B,C). Notably, W99, present in this region, shows doubling of the indole proton. Loop II, the region where most of the resonance splitting is observed, is also involved in small ligand binding (PDB ID 4EAX and 4XPJ). Peak doubling was furthermore observed for the side chains of a few residues in the ${ }^{13} \mathrm{C} \mathrm{HCCH}-\mathrm{TOCSY}$ and ${ }^{13} \mathrm{C}$ NOESYHSQC spectra (data not shown). To test for the presence of conformational exchange, ${ }^{15} \mathrm{~N}$ HSQC spectra were recorded at $10^{\circ} \mathrm{C}$ intervals between $10^{\circ} \mathrm{C}$ and $40^{\circ} \mathrm{C}$ (Figure 2A). At $10^{\circ} \mathrm{C}$, the peak intensity is highly heterogeneous with only ca. $30 \%$ of the number expected for a homodimer of 118 amino acids. By progressively increasing the temperature to $40^{\circ} \mathrm{C}$, all the expected signals return, with the vast majority showing homogeneous intensity. However, a subset of secondary species of lower intensity remained observable. The overall quality of the spectrum is consistent with that expected for a homodimer of $26 \mathrm{kDa}$, with a line width indicative of the molecular size of the system. For a homodimeric protein, resonance doubling can be explained in at least two possible ways. It could indicate an intrinsic local asymmetry of the homodimer over the NMR timescale. This possibility can, however, be ruled out since the most affected region around loop II is not engaged in the homodimer interface. Alternatively, it could arise from the presence of multiple conformational species in equilibrium on the slow-exchange timescale. This second possibility seems more likely given the dramatic change in the number and in the relative peak intensities observed in the ${ }^{15} \mathrm{~N}$ HSQC as a function of temperature.

The data thus suggest that the NGF loops adopt different conformations in the slow exchange regime in the NMR timeframe.

\section{Dynamical Features of the NGF loops}

We measured the T1, T2 and heteronuclear NOE (hetNOE) parameters to obtain information on the dynamics in solution. The experimental correlation time $\left(\mathrm{T}_{\mathrm{C}}\right)$ is $14.8 \mathrm{~ns}$ which is consistent with the expected molecular weight of the dimer. The T1, T2, and NOE values correlate well with the secondary structure (Figure 3A) and highlight the more rigid $\beta$-sheet regions. The loops, especially II and $\mathrm{V}$, and the $\mathrm{C}$-terminus are relatively more flexible showing hetNOE values lower than the average (0.7). However, overall, the hetNOE profile is relatively flat with some minor exceptions which could well be explained by residue overlap.

Intrigued by these results, we ran a molecular dynamics simulation of mNGF (200 ns) to further explore the tendency of the N-terminus to adopt a helical conformation and the conformational space of the loop regions. The ensemble of structures sampled in the MD trajectory is variable, but in line with the NMR analysis (Figure 3B). Not having imposed any symmetry constraints we see of course a different behavior in
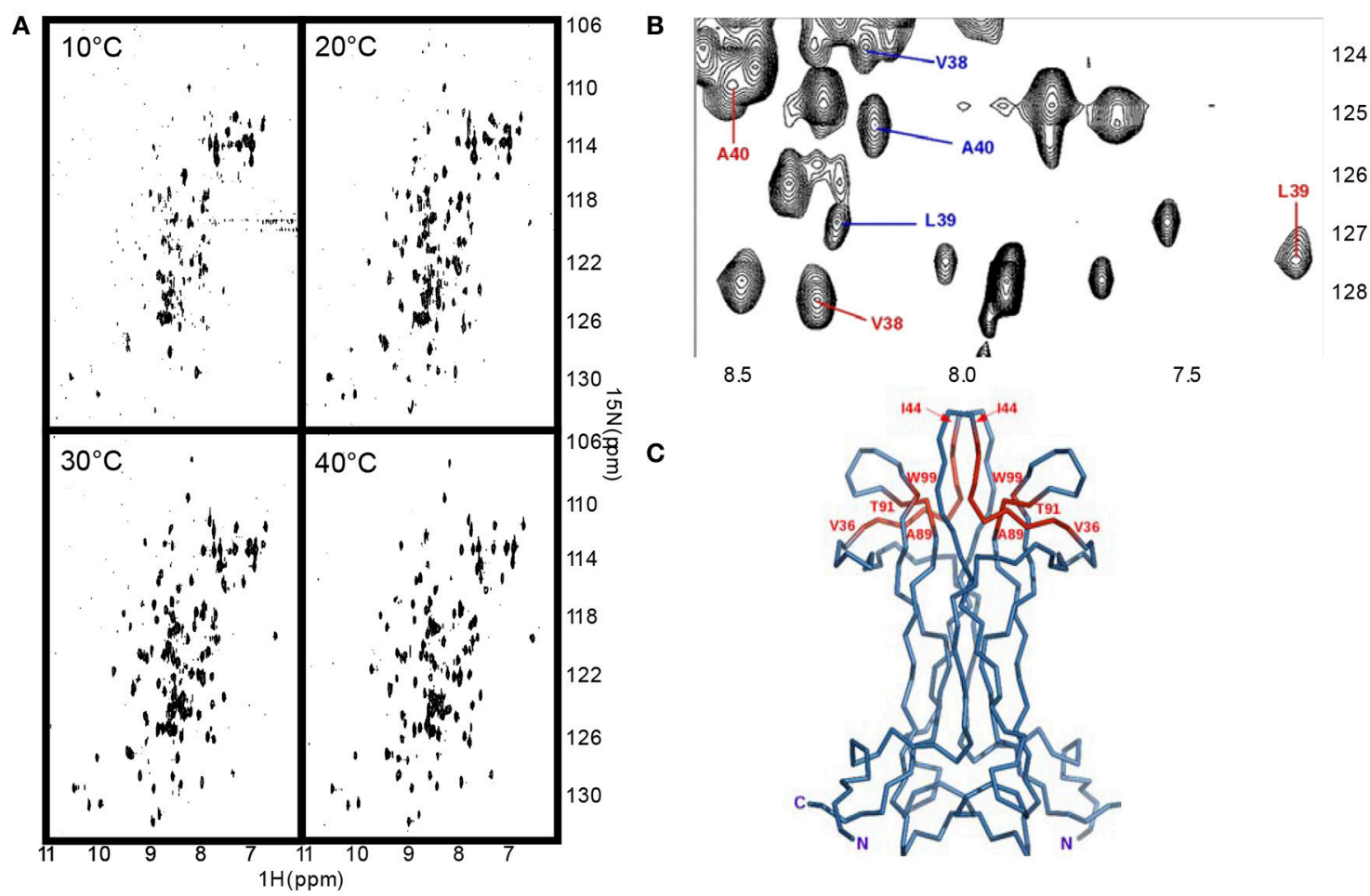

8.5

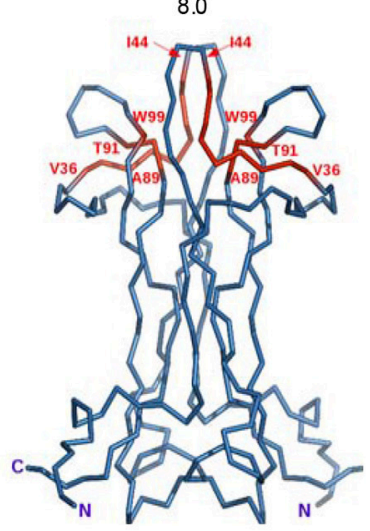

7.5

FIGURE 2 | NGF is in a conformational exchange regime. (A) Effect of a temperature scan from 10 to $30^{\circ} \mathrm{C}$ on the $15 \mathrm{~N} \mathrm{HSQC} \mathrm{spectra} \mathrm{of} \mathrm{NGF.} \mathrm{(B)} \mathrm{Close-up} \mathrm{of}$ one of the regions of the $2 \mathrm{D} 1 \mathrm{H}-15 \mathrm{~N}-\mathrm{HSQC}$ at $30^{\circ} \mathrm{C}$ showing the presence of double species. In blue the main species, in red the secondary ones. (C) Mapping on NGF structure (blue) of the residues showing a double species in the spectra (red). The residues where peak splitting was observed were: V36-144, A89-T91, W99 (amidic indole only). 


\section{A}
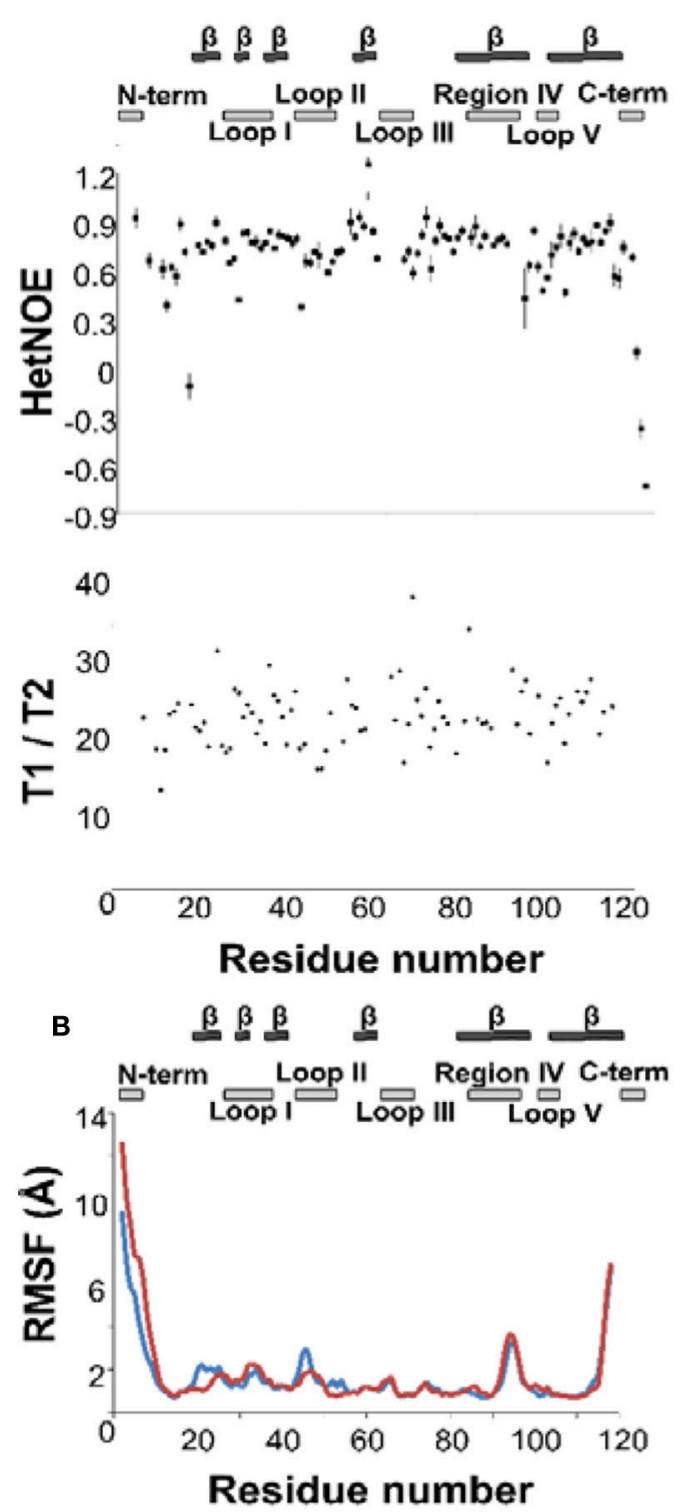
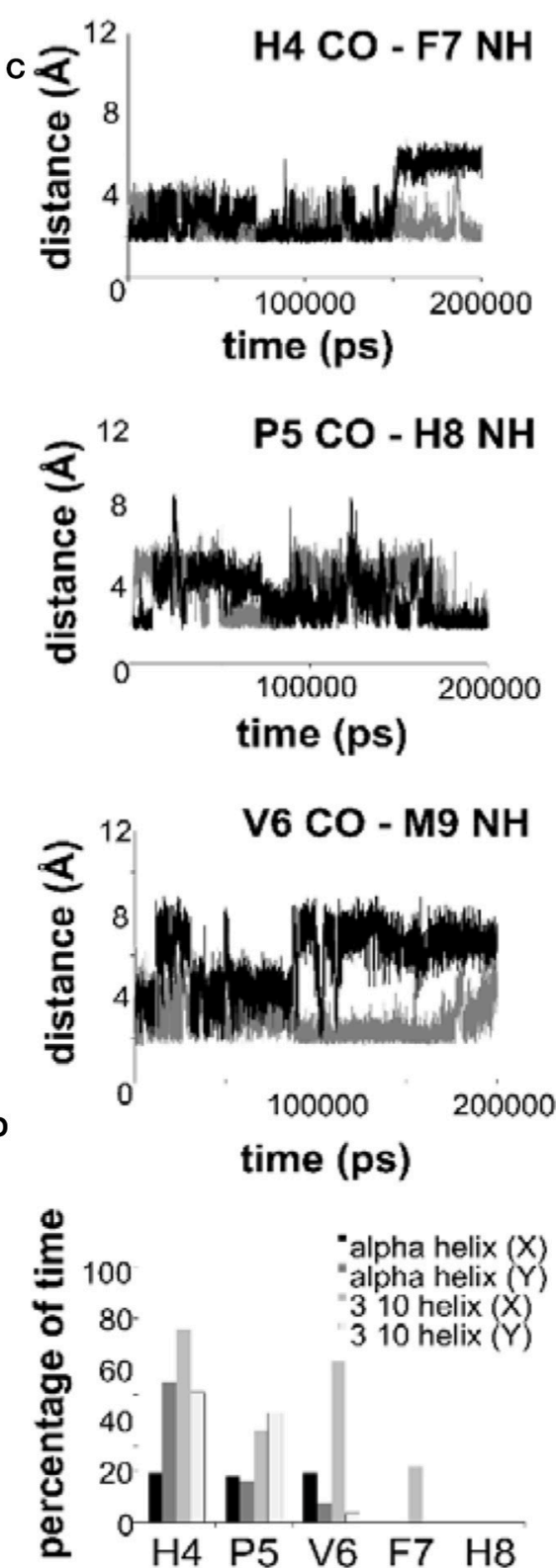

FIGURE 3 | The dynamics of NGF in solution. (A) ${ }^{15} \mathrm{~N}-\mathrm{NMR}$ relaxation studies. Plots of the T1/T2 and $15 \mathrm{~N}-1 \mathrm{H}$ NOE of $\mathrm{mNGF}$ at $600 \mathrm{MHz}$ and $30^{\circ} \mathrm{C}$. The secondary structure is shown above. (B) R.m.s.d. fluctuations along the $200 \mathrm{~ns}$ MD simulation of NGF vs. residue position for the two protomers (blue- protomer x; red-protomer y). (C) Evolution of the distances $(\AA)$, along the 200 ns MD simulation, between atoms engaged in the hydrogen bonds that stabilize the helical structure of the N-terminus of mNGF (gray-protomer $\mathrm{x}$; black-protomer y). (D) Percentage of time, along the 200 ns MD simulation, that each residue spent in a helical conformation along the trajectory.

the two protomers. Over the simulation time scale, all residues in the tract His4-Phe7 spend a significant percentage of time in a 310 or $\alpha$-helix conformation at least in one of the protomers (Figure 3C). Residues H4-G10, in both protomers, unwind and fold during the simulation but are able to form transient hydrogen bonds mostly between the carbonyl oxygen of $\mathrm{H} 4$ and the amide hydrogen of F7 and the carbonyl oxygen of P5 and the amide hydrogen of H8. The carbonyl oxygen of V6 is also engaged only in one protomer in hydrogen bonds with the amide hydrogen of either M9 or G10 residues. The mainchain-side-chain interactions between residues H4/F7, V6/M9, and F7/G10 are overall more preserved than the side-chainside-chain interactions between residues $\mathrm{P} 5 / \mathrm{H} 8$ and V6/M9 as well as the interaction between the residues V6/G10. The MD results are in line with the observed NOE contacts supporting that in at least one of the protomers (Table S1), a short 
segment within the central region of the mNGF $\mathrm{N}$-terminus adopts a $\alpha$-helix (15\%) or a 310 -helix (35\%) conformation for a small but significant fraction of time (Figure 3D). According to these conclusions, a previous MD study on an isolated linear peptide spanning the $\mathrm{N}$-terminal sequence (Stanzione et al., 2010) has shown that most of the adopted conformations do not present a regular secondary structure but a helical structure can form for a limited fraction of conformers in the ensemble.

We can thus conclude that the NGF N-terminus comprises a nascent helix which can be further stabilized upon binding with its partners.

\section{Analysis of the Loop Motions}

The $\mathrm{C}_{\alpha}$ r.m.s.d. along the MD trajectory shows that, besides the $\mathrm{N}$ - and C-termini, the regions encompassing loops II and V are comparably more flexible and exhibit large-scale conformational flexibility (Figure 3B). The loop variations are however relatively small as compared to the flexibility of the $\mathrm{N}$ - and C-termini. This indicates that the loops are plastic but not flexible in agreement with the differences observed in these regions in the crystallographic structures of apo mNGF (PDB ID 1BET, 1BTG) and in complex with lysophosphatidylserine (PDB ID 4EAX) and lysophosphatidylinositol (PDB ID 4XPJ). This information is of crucial importance for the development of small organic compounds (Brahimi et al., 2014) and peptidomimetics based on the combination of different NGF loop fragments (Colangelo et al., 2008). We thus analyzed the relative intra- and interprotomer distances between loops I, II, III, and V (Figure S1). The shortest and the longest inter-protomer $\mathrm{C}_{\alpha}$ distances between the Asn 46 of Loop II are $11 \AA$ (apo form PDB ID 1BTG, protomers B,C) and $30 \AA$ respectively (holo forms PDB ID 4XPJ, protomers $\mathrm{A}, \mathrm{B}$ and $\mathrm{PDB}$ ID $4 \mathrm{EAX}$, protomers $\mathrm{A}, \mathrm{B}$ and $\mathrm{C}, \mathrm{D}$ ) indicating large scale motions. The corresponding distances, in the most representative MD structure (the most populated cluster center) and in the lowest global energy NMR structure are 13 and $16 \AA$ respectively. The longest and shortest interprotomers distances between Lys 95 of Loop V are $42 \AA$ (apo form PDB ID 1BTG, protomers B,C) and $40 \AA$ (holo form PDB ID 4XPJ). The corresponding distances in the most representative MD structure (the most populated cluster center) and in the lowest global energy NMR structure are 34 and $37 \AA$ respectively (Figure S1).

For comparison, in the crystal structures of free and bound NGF, the conformation of loop II has high plasticity with an overall opening of the structure upon the accommodation of the small ligands (Figure 1C). It is tempting to speculate that the conformational equilibrium experienced by loop II and V, supported also by resonance doubling, is functionally relevant for the biological activity of the molecule, including interactions with the binding partners, i.e. the receptors TrkA and $\mathrm{p} 75^{\mathrm{NTR}}$. These features highlight the differences between NGF and other homodimeric $\beta$-sandwich structures.

We can thus conclude that NGF has long plastic but relatively rigid loops. This information is of crucial importance for drug design (Bannwarth and Kostine, 2014).

\section{Using the Spectral Assignment for Antibody Recognition Studies}

Finally, we used the information gained in solution to study the interaction of NGF with $\alpha \mathrm{D} 11$, one of the well-characterized antibodies. We titrated ${ }^{15} \mathrm{~N}$-labeled NGF with $\alpha \mathrm{D} 11$ using the $\mathrm{MAb}$ protein. We used sub-stoichiometric amounts of $\alpha \mathrm{D} 11$ to allow the observation of shift changes, given that the high affinity expected and the large molecular weight of the complex could prevent observation of the signals at stoichiometric concentrations (Figure 4A). The most appreciable perturbations in the NGF spectrum occur in three distinct sets of resonances which disappear (V14, G23, F101, C110, V111), decrease in intensity (T26, L39, N45, N46, Y52, E65, D93, W99, R100, K115) or shift significantly (G10, S13, V18, A40, F49, T56, Y79, T81, T92, E94, K95, Q96, I102, V109) indicating slow, slowintermediate and fast-exchange regimes, respectively. When mapping these regions onto the NGF structure, a set of the affected residues (V18, G23, T26, L39, A40, F49, Y52, D93, Q96, W99, R100, F101) overlap with those previously identified in the SAXS hNGF- $\alpha$ D11 FAb complex model (Covaceuszach et al., 2008). Additionally, residues G10-V14, V64-I71, T81, and V109V111, which cluster close together in space, suggest a previously unidentified epitope (Figure 4B). Interestingly, this region is not included in the FAb-NGF contacts in the crystal structures of the complexes of hNGF with the anti-NGF antibodies Tanezumab (PDB ID 4EDW) and its murine precursor MAb 911 (PDB ID $4 \mathrm{EDX}$ ), whose epitopes are otherwise comparable to those of $\alpha \mathrm{D} 11$ FAb. Thus, the solution NMR epitope mapping of the MAb $\alpha$ D11 unveiled a previously undetected epitope, further supporting why $\alpha \mathrm{D} 11$ antibody binding differs significantly between mature NGF and proNGF: in the latter the propeptide could hinder the accessibility of the NGF surface to the antibody.

\section{DISCUSSION}

NGF is an important molecule involved in the maintenance of numerous neuronal populations, and its alterations in metabolism are crucial for different neurological disorders. The crystal structures of mNGF have been known for 25 years (McDonald et al., 1991; Holland et al., 1994), but no solution structure has so far been available which could facilitate drug design and small molecule screening in solution. The present study fills this gap, by reporting the mNGF structure in solution and sheds light on local conformational and dynamical features supported by MD simulations.

For the first time we have experimentally characterized the $\mathrm{N}$-terminus of the protein, a dynamic and elusive segment of NGF which was previously only observed in the structure of recombinant hNGF in complex with TrkA receptor while it is cleaved or unobservable in other structures. This region is considered a structural determinant in NGF molecular recognition and selective binding of the TrkA rather than $\mathrm{p} 75^{\mathrm{NTR}}$ receptor (Wiesmann et al., 1999; He and Garcia, 2004; Wehrman et al., 2007). Our data indicate that, in the context of the full length mNGF, a helical conformation is readily sampled in 


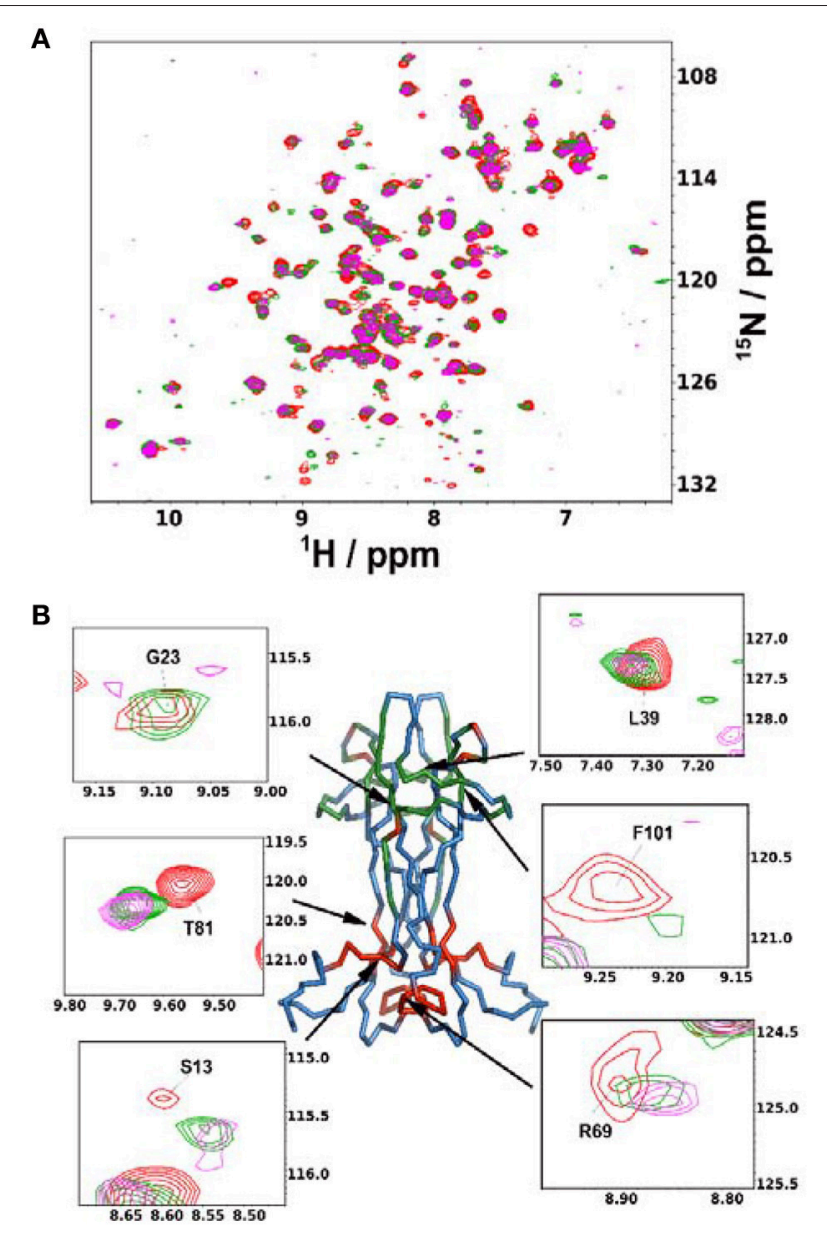

FIGURE 4 | Anti-NGF Antibody-antigen interaction. (A) Superimposition of the ${ }^{15} \mathrm{~N}$-HSQC recorded at the different points of the titration of $\mathrm{mNGF}$ with the MAb $\alpha \mathrm{D} 11$. The spectra were recorded at $30^{\circ} \mathrm{C}$. Red: 0 antibody equivalents; Green: 0.15 antibody equivalents; Purple: 0.45 antibody equivalents. (B) Structure of $\mathrm{mNGF}$ (PDB ID 1BET) with the residues of the $\alpha \mathrm{D} 11$ epitope highlighted in green. Residues found to be affected in the present titration but not previously known to participate to the epitope are highlighted in red. Insets: Close-ups of the residues most affected by broadening or chemical shift perturbation. Their positions on the structure is indicated by arrows.

solution among the energetically favored states of this region as in a classical nascent helix. This result challenges the current view that the NGF N-terminus is unstructured and adopts a helical structure only upon binding to its partner TrkA (Wehrman et al., 2007). Our findings open up new strategies for the development of effective NGF N-terminal based bioactive peptide-based compounds as antagonists with high-affinity toward the TrkA receptor.

From a careful analysis of the resonances in the ${ }^{15} \mathrm{~N}$ - and ${ }^{13} \mathrm{C}-\mathrm{HSQC}$ and the corresponding NOESY-HSQCs, it was also possible to identify the splitting of several resonances. The residues involved are all concentrated in the region of the molecule encompassing loops I and II and the facing region IV/loop V. The occurrence of these extra resonances likely results from a slow exchange between conformational species, thus pointing to an increased mobility of the loops II and $\mathrm{V}$ within the frame of an otherwise rigid molecule. Accordingly, these regions have relatively lower values of hetNOEs and a higher r.m.s.d. in our MD trajectory although overall the motions look only local. These observations agree with the structural information available for other members of the neurotrophin family (Butte et al., 1998; Robinson et al., 1999; Banfield et al., 2001; Gong et al., 2008) and with the observation that the region encompassing loops $\mathrm{II}$ and $\mathrm{V}$ is the one in which a conformational rearrangement/breathing is triggered by binding of lysophospholipids (Tong et al., 2012; Sun and Jiang, 2015). We can rationalize these results considering that loops II and $\mathrm{V}$ can be considered like forceps that open/close and are in a cross-talk to adapt and sequester the ligand. Loops I are spatially close to loops V but do not move. They could thus be considered like a "guard rail" preventing excessive motions of loops $\mathrm{V}$ and consequent disruption of the binding site formed by loops II and V. Loop III protects the cystine-knot. It is spatially far from loops I, II and V, but in proximity of the $\mathrm{N}$ - and $\mathrm{C}$ termini. It is not flexible in agreement with its likely role of protection even though it is peculiar that this loop has partially defined electronic density in the crystallographic structures of human NGF in complex with the TrkA and $\mathrm{p} 75^{\mathrm{NTR}}$ receptors, as well as in complex with the DNA-aptamer and with antibodies and it is among the least conserved in length and composition within the neurotrophin family members (NGF, BDNF, NT-3, NT-4).

Finally, the present NMR study exploits the interaction between NGF and the MAb $\alpha \mathrm{D} 11$ antibody allowing a fine mapping of the epitope: We observed a patch encompassing residues G10-V14, V64-I71, T81, and V109-V111, as being involved in addition to loop I and II which had already been identified (Covaceuszach et al., 2008). These regions face each other in the structure. The observation is important for the interpretation of the binding properties of $\alpha \mathrm{D} 11$ which recognizes both mature and proNGF albeit with very different affinities (pM vs. nM). Since $\alpha \mathrm{D} 11$ is an NGF neutralizing antagonistic antibody (Covaceuszach et al., 2008), knowledge of the exact epitopes is of interest for interpreting mouse models and for the design of more effective antibodies.

In conclusion, our study reports the first NMR study of mNGF in solution, and, more generally, of a neurotrophin and provides new insights into the dynamical features of NGF N-terminus and loops, opening the way to further studies aimed at a deeper understanding of NGF binding to its partners. It also paves the way to the determination of the high resolution structure of the mNGF precursor, mproNGF, whose only available 3D structural information is limited to low resolution SAXS studies (Paoletti et al., 2009) and preliminary solution NMR studies (Paoletti et al., 2011).

\section{Experimental Procedures \\ Expression, Refolding, and Purification of mNGF}

Recombinant mNGF was expressed in minimal medium, both as ${ }^{15} \mathrm{~N}$ and a ${ }^{15} \mathrm{~N}-{ }^{13} \mathrm{C}$ labeled proteins, and purified as previously 
described (Paoletti et al., 2011). See Supplementary Information for details.

\section{NMR Spectroscopy Experiments}

NMR experiments and structure calculations were carried out using standard protocols (details in Supplementary Information). Briefly, HNCA, HNCO, HNCACB were used for backbone assignments. ${ }^{15} \mathrm{~N}$ NOESY-HSQC, ${ }^{13} \mathrm{C}$ NOESY-HSQC, CBCACONH, and HCCH-TOCSY were used for side chain aliphatic assignments. $(\mathrm{H} \beta) \mathrm{C} \beta(\mathrm{C} \gamma \mathrm{C} \delta) \mathrm{H} \delta$ and $(\mathrm{H} \beta) \mathrm{C} \beta(\mathrm{C} \gamma \mathrm{C} \delta) \mathrm{H} \varepsilon$ were used in combination with ${ }^{13} \mathrm{C}$-HSQC, ${ }^{13} \mathrm{C}$ - NOESY-HSQC, and $\mathrm{HCCH}$-TOCSY tuned for the aromatic resonances for the assignment of the aromatic side chains. All spectra were processed using NMRPipe/NMR-Draw (Delaglio et al., 1995) and analyzed using CARA (Keller, 2004). Backbone and side chain assignment was deposited in the BMRB database (accession code 34037).

Relaxation parameters were obtained by spectra recorded at $600 \mathrm{MHz}$ and $30^{\circ} \mathrm{C}$ and extracted using peak picking, lineshape fitting, and exponential modeling as implemented in NMRPipe (Delaglio et al., 1995).

\section{Structure Determination}

Automated NOESY cross-peak assignments and structure determination were performed using the ARIA 2.3 software (Rieping et al., 2007). The input used to generate the final structures consisted of NGF intra-molecular NOE cross peaks from ${ }^{15} \mathrm{~N}$ - and ${ }^{13} \mathrm{C}$-NOESY-HSQC spectra (at 30 and $35^{\circ} \mathrm{C}$ ), along with a set of $\varphi$ and $\psi$ backbone dihedral restraints derived by TALOS+ (Shen et al., 2009) and a set of manually assigned unambiguous inter-protomer restraints. After refinement of the 60 lowest global energy structures by molecular dynamics simulation with explicit water, 20 structures ranked on global energy were selected as representative of the structure and used for statistical analysis. Structure quality was evaluated with PROCHECK-NMR (Laskowski et al., 1996). The details are reported in the Supplementary Information.

The coordinates of the NGF structure are deposited in the PDB under the code 5LSD.

\section{Molecular Dynamics Simulations and Analysis}

A molecular model of the full length mNGF encompassing residues (2-118) was built (Covaceuszach et al., 2015) from

\section{REFERENCES}

Banfield, M. J., Naylor, R. L., Robertson, A. G., Allen, S. J., Dawbarn, D., and Brady, R. L. (2001). Specificity in Trk receptor:neurotrophin interactions: the crystal structure of TrkB-d5 in complex with neurotrophin-4/5. Structure 9, 1191-1199. doi: 10.1016/S0969-2126(01)00681-5

Bannwarth, B., and Kostine, M. (2014). Targeting nerve growth factor (NGF) for pain management: what does the future hold for NGF antagonists? Drugs 74, 619-626. doi: 10.1007/s40265-014-0208-6

Berrera, M., Cattaneo, A., and Carloni, P. (2006). Molecular simulation of the binding of nerve growth factor peptide mimics to the receptor tyrosine kinase A. Biophys. J. 91, 2063-2071. doi: 10.1529/biophysj.106.083519

Brahimi, F., Ko, E., Malakhov, A., Burgess, K., and Saragovi, H. U. (2014). Combinatorial assembly of small molecules into bivalent antagonists of the crystal structures of mouse bis-des-octa $\beta$-NGF (PDB ID 1BTG, protomers B,C). Molecular dynamics simulation (MD) was performed using the GROMACS software package (version 5.1.2) (Hess et al., 2008) conjugated with the Amber99SB force field. Details are reported in the Supplementary Experimental procedures. The utilities gmx distance and gmx cluster, provided in the GROMACS package, were used: (i) to calculate the distances between pairs of positions as a function of time; (ii) to cluster, in the post processing phase, the resulting trajectories with a cutoff of $0.15 \mathrm{~nm}$, calculated on the backbone atoms (Daura et al., 1999).

\section{AUTHOR CONTRIBUTIONS}

FP, CdC, DL, AP, AC conceived and designed the experiments; FP, CdC, FM, RY, SC, GK conducted the experiments and analyzed the data; FP, CdC, DL, AP, AC interpreted the data and wrote the paper.

\section{FUNDING}

The research described here was funded by the European Community's Seventh Framework Program Paincage Grant $\mathrm{Nr}$ 603191, MRC (U117584256), and MIUR, project PRIN \#2010N8PBAA_006. FP was recipient of a Royal SocietyAccademia dei Lincei Fellowship.

\section{ACKNOWLEDGMENTS}

We gratefully acknowledge the help of Dr. Giuseppe Nicastro (The Francis Crick Institute) for recording a set of NMR data and for helpful discussion, the assistance of Dr. Andrea Delise (SISSA) with the software managing and the helpful suggestions of Dr. Benjamin Bardiaux (Inst. Pasteur, Paris) for the ARIA software usage.

\section{SUPPLEMENTARY MATERIAL}

The Supplementary Material for this article can be found online at: http://journal.frontiersin.org/article/10.3389/fmolb. 2016.00083/full\#supplementary-material
TrkC or TrkA receptors. PLoS ONE 9:e89617. doi: 10.1371/journal.pone.00 89617

Butte, M. J., Hwang, P. K., Mobley, W. C., and Fletterick, R. J. (1998). Crystal structure of neurotrophin-3 homodimer shows distinct regions are used to bind its receptors. Biochemistry 37, 16846-16852. doi: 10.1021/bi98 $1254 \mathrm{o}$

Capsoni, S. (2014). From genes to pain: nerve growth factor and hereditary sensory and autonomic neuropathy type V. Eur. J. Neurosci. 39, 392-400. doi: 10.1111/ejn.12461

Capsoni, S., Brandi, R., Arisi, I., D’Onofrio, M., and Cattaneo, A. (2011). A dual mechanism linking NGF/proNGF imbalance and early inflammation to Alzheimer's disease neurodegeneration in the AD11 anti-NGF mouse model. CNS Neurol. Disord. Drug Targets 10, 635-647. doi: $10.2174 / 187152711796235032$ 
Capsoni, S., and Cattaneo, A. (2006). On the molecular basis linking Nerve Growth Factor (NGF) to Alzheimer's disease. Cell. Mol. Neurobiol. 26, 619-633. doi: 10.1007/s10571-006-9112-2

Cattaneo, A., Rapposelli, B., and Calissano, P. (1988). Three distinct types of monoclonal antibodies after long-term immunization of rats with mouse nerve growth factor. J. Neurochem. 50, 1003-1010. doi: 10.1111/j.1471-4159.1988.tb10565.x

Colangelo, A. M., Bianco, M. R., Vitagliano, L., Cavaliere, C., Cirillo, G., De Gioia, L., et al. (2008). A new nerve growth factor-mimetic peptide active on neuropathic pain in rats. J. Neurosci. 28, 2698-2709. doi: 10.1523/JNEUROSCI.5201-07.2008

Covaceuszach, S., Cassetta, A., Konarev, P. V., Gonfloni, S., Rudolph, R., Svergun, D. I., et al. (2008). Dissecting NGF interactions with TrkA and p75 receptors by structural and functional studies of an anti-NGF neutralizing antibody. J. Mol. Biol. 381, 881-896. doi: 10.1016/j.jmb.2008.06.008

Covaceuszach, S., Konarev, P. V., Cassetta, A., Paoletti, F., Svergun, D. I., Lamba, D., et al. (2015). The conundrum of the high-affinity NGF binding site formation unveiled? Biophys. J. 108, 687-697. doi: 10.1016/j.bpj.2014.11.3485

Covaceuszach, S., Marinelli, S., Krastanova, I., Ugolini, G., Pavone, F., Lamba, D., et al. (2012). Single cycle structure-based humanization of an anti-nerve growth factor therapeutic antibody. PLOS ONE 7:e32212. doi: 10.1371/journal.pone.0032212

Daura, X., Gademann, K., Jaun, B., Seebach, D., van Gunsteren, W. F., and Mark, A. E. (1999). Peptide folding: when simulation meets experiment. Angew. Chem. Int. Ed. 38, 236-240. doi: $\quad 10.1002 /($ SICI)1521-3773(19990115)38:1/2<236::AID-ANIE236>3.0. $\mathrm{CO} ; 2-\mathrm{M}$

Delaglio, F., Grzesiek, S., Vuister, G. W., Zhu, G., Pfeifer, J., and Bax, A. (1995). NMRPipe: a multidimensional spectral processing system based on UNIX pipes. J. Biomol. NMR 6, 277-293. doi: 10.1007/BF00197809

Feng, D., Kim, T., Ozkan, E., Light, M., Torkin, R., Teng, K. K., et al. (2010). Molecular and structural insight into proNGF engagement of p75NTR and sortilin. J. Mol. Biol. 396, 967-984. doi: 10.1016/j.jmb.2009.12.030

Gong, Y., Cao, P., Yu, H. J., and Jiang, T. (2008). Crystal structure of the neurotrophin-3 and p75NTR symmetrical complex. Nature 454, 789-793. doi: $10.1038 /$ nature 07089

He, X.-L., and Garcia, K. C. (2004). Structure of nerve growth factor complexed with the shared neurotrophin receptor p75. Science 304, 870-875. doi: $10.1126 /$ science. 1095190

Hempstead, B. L. (2014). Deciphering proneurotrophin actions. Handb. Exp. Pharmacol. 220, 17-32. doi: 10.1007/978-3-642-45106-5_2

Hess, B., Kutzner, C., van der Spoel, D., and Lindahl, E. (2008). GROMACS 4: algorithms for highly efficient, load-balanced, and scalable molecular simulation. J. Chem. Theory Comput. 4, 435-447. doi: 10.1021/ct700301q

Holland, D. R., Cousens, L. S., Meng, W., and Matthews, B. W. (1994). Nerve growth factor in different crystal forms displays structural flexibility and reveals zinc binding sites. J. Mol. Biol. 239, 385-400. doi: 10.1006/jmbi.199 4.1380

Hughes, A. L., Messineo-Jones, D., Lad, S. P., and Neet, K. E. (2001). Distinction between differentiation, cell cycle, and apoptosis signals in PC12 cells by the nerve growth factor mutant delta9/13, which is selective for the p75 neurotrophin receptor. J. Neurosci. Res. 63, 10-19. doi: 10.1002/1097-4547(20010101)63:1<10::AID-JNR2>3.0.CO;2-R

Jarvis, T. C., Davies, D. R., Hisaminato, A., Resnicow, D. I., Gupta, S., Waugh, S. M., et al. (2015). Non-helical dna triplex forms a unique aptamer scaffold for high affinity recognition of nerve growth factor. Structure 23, 1293-1304. doi: 10.1016/j.str.2015.03.027

Kahle, P., Burton, L. E., Schmelzer, C. H., and Hertel, C. (1992). The amino terminus of nerve growth factor is involved in the interaction with the receptor tyrosine kinase p140trkA. J. Biol. Chem. 267, 22707-22710.

Kelleher, J. H., Tewari, D., and McMahon, S. B. (2017). Neurotrophic factors and their inhibitors in chronic pain treatment. Neurobiol. Dis. 97(Pt B), 127-138. doi: $10.1016 /$ j.nbd.2016.03.025

Keller, R. (2004). The Computer Aided Resonance Assignment Tutorial. Goldau: Cantina Verlag.

La Porte, S. L., Eigenbrot, C., Ultsch, M., Ho, W.-H., Foletti, D., Forgie, A., et al. (2014). Generation of a high-fidelity antibody against nerve growth factor using library scanning mutagenesis and validation with structures of the initial and optimized Fab-antigen complexes. mAbs 6, 1059-1068. doi: $10.4161 /$ mabs. 28677

Laskowski, R. A., Rullmannn, J. A., MacArthur, M. W., Kaptein, R., and Thornton, J. M. (1996). AQUA and PROCHECK-NMR: programs for checking the quality of protein structures solved by NMR. J. Biomol. NMR 8, 477-486. doi: $10.1007 / \mathrm{BF} 00228148$

Levi-Montalcini, R. (1987). The nerve growth factor 35 years later. Science 237, 1154-1162. doi: 10.1126/science.3306916

Lewin, G. R., and Carter, B. D. (2014). Neurotrophic Factors. Berlin; Heidelberg: Springer.

McDonald, N. Q., Lapatto, R., Murray-Rust, J., Gunning, J., Wlodawer, A., and Blundell, T. L. (1991). New protein fold revealed by a 2.3-A resolution crystal structure of nerve growth factor. Nature 354, 411-414. doi: 10.1038/354 $411 \mathrm{a} 0$

Mysona, B. A., Matragoon, S., Stephens, M., Mohamed, I. N., Farooq, A., Bartasis, M. L., et al. (2015). Imbalance of the nerve growth factor and its precursor as a potential biomarker for diabetic retinopathy. BioMed. Res. Int. 2015:571456. doi: $10.1155 / 2015 / 571456$

Nilges, M., and O’Donoghue, S. I. (1998). Ambiguous NOEs and automated NOE assignment. Prog. Nucl. Magn. Reson. Spectrosc. 32, 107-139. doi: 10.1016/S0079-6565(97)00025-3

Paoletti, F., Covaceuszach, S., Konarev, P. V., Gonfloni, S., Malerba, F., Schwarz, E., et al. (2009). Intrinsic structural disorder of mouse proNGF. Proteins 75, 990-1009. doi: 10.1002/prot.22311

Paoletti, F., Malerba, F., Kelly, G., Noinville, S., Lamba, D., Cattaneo, A., et al. (2011). Conformational plasticity of proNGF. PLoS ONE 6:e22615. doi: 10.1371/journal.pone.0022615

Rieping, W., Habeck, M., Bardiaux, B., Bernard, A., Malliavin, T. E., and Nilges, M. (2007). ARIA2: automated NOE assignment and data integration in NMR structure calculation. Bioinformatics 23, 381-382. doi: 10.1093/bioinformatics/btl589

Robinson, R. C., Radziejewski, C., Spraggon, G., Greenwald, J., Kostura, M. R., Burtnick, L. D., et al. (1999). The structures of the neurotrophin 4 homodimer and the brain-derived neurotrophic factor/neurotrophin 4 heterodimer reveal a common Trk-binding site. Protein Sci. 8, 2589-2597. doi: 10.1110/ps.8. 12.2589

Saudek, V., Pastore, A., Morelli, M. A., Frank, R., Gausepohl, H., and Gibson, T. (1991). The solution structure of a leucine-zipper motif peptide. Protein Eng. 4, 519-529. doi: 10.1093/protein/4.5.519

Settanni, G., Cattaneo, A., and Carloni, P. (2003). Molecular dynamics simulations of the NGF-TrkA domain 5 complex and comparison with biological data. Biophys. J. 84, 2282-2292. doi: 10.1016/S0006-3495(03)75034-6

Shen, Y., Delaglio, F., Cornilescu, G., and Bax, A. (2009). TALOS+: a hybrid method for predicting protein backbone torsion angles from NMR chemical shifts. J. Biomol. NMR 44, 213-223. doi: 10.1007/s10858-0099333-z

Stanzione, F., Esposito, L., Paladino, A., Pedone, C., Morelli, G., and Vitagliano, L. (2010). Role of the conformational versatility of the neurotrophin N-terminal regions in their recognition by Trk receptors. Biophys. J. 99, 2273-2278. doi: 10.1016/j.bpj.2010.07.054

Sun, H. L., and Jiang, T. (2015). The structure of nerve growth factor in complex with lysophosphatidylinositol. Acta Crystallogr. F Struct. Biol. Commun. 71, 906-912. doi: 10.1107/S2053230X15008870

Tiveron, C., Fasulo, L., Capsoni, S., Malerba, F., Marinelli, S., Paoletti, F., et al. (2013). ProNGF NGF imbalance triggers learning and memory deficits, neurodegeneration and spontaneous epileptic-like discharges in transgenic mice. Cell Death Differ. 20, 1017-1030. doi: 10.1038/cdd. 2013.22

Tong, Q., Wang, F., Zhou, H.-Z., Sun, H.-L., Song, H., Shu, Y.-Y., et al. (2012). Structural and functional insights into lipid-bound nerve growth factors. FASEB J. 26, 3811-3821. doi: 10.1096/fj.12-207316

Travaglia, A., Pietropaolo, A., Di Martino, R., Nicoletti, V. G., La Mendola, D., Calissano, P., et al. (2015). A small linear peptide encompassing the NGF N-terminus partly mimics the biological activities of the entire neurotrophin in PC12 cells. ACS Chem. Neurosci. 6, 1379-1392. doi: 10.1021/acschemneuro.5b00069 
Wehrman, T., He, X., Raab, B., Dukipatti, A., Blau, H., and Garcia, K. C. (2007). Structural and mechanistic insights into nerve growth factor interactions with the TrkA and p75 receptors. Neuron 53, 25-38. doi: 10.1016/j.neuron.2006.09.034

Wiesmann, C., Ultsch, M. H., Bass, S. H., and de Vos, A. M. (1999). Crystal structure of nerve growth factor in complex with the ligand-binding domain of the TrkA receptor. Nature 401, 184-188. doi: 10.1038/43705

Woo, S. B., Timm, D. E., and Neet, K. E. (1995). Alteration of NH2terminal residues of nerve growth factor affects activity and Trk binding without affecting stability or conformation. J. Biol. Chem. 270, 6278-6285. doi: $10.1074 / j b c .270 .11 .6278$
Conflict of Interest Statement: The authors declare that the research was conducted in the absence of any commercial or financial relationships that could be construed as a potential conflict of interest.

Copyright (® 2016 Paoletti, de Chiara, Kelly, Covaceuszach, Malerba, Yan, Lamba, Cattaneo and Pastore. This is an open-access article distributed under the terms of the Creative Commons Attribution License (CC BY). The use, distribution or reproduction in other forums is permitted, provided the original author(s) or licensor are credited and that the original publication in this journal is cited, in accordance with accepted academic practice. No use, distribution or reproduction is permitted which does not comply with these terms. 